\title{
A Comfortable Palatal Delayed Surgical Obturator for Hemimaxillectomy Patients
}

\author{
Paudel SP ${ }^{1}$, Paudel S $\mathrm{S}^{2}$, Dhungana $\mathrm{M}^{3}$, Shrestha $\mathrm{P}^{4 *}$ \\ ${ }^{1}$ Dental Surgeon, Lumbini Zonal Hospital, Butwal, Nepal \\ ${ }^{2,3}$ Lecturer, ${ }^{4}$ Associate Professor, Department of Prosthodontics and Maxillofacial Prosthetics, \\ KIST Medical College, Lalitpur, Nepal
}

\begin{abstract}
A surgical obturator prosthesis is commonly used as an effective means for rehabilitating patient with hemi-maxillectomy defect. The obturator supports the soft tissues after surgery and minimizes the contraction of scar and disfigurement thereby making a positive effect on patient's overall function and psychology. The goal of prosthodontics to restore missing orofacial structures for improving facial esthetics and oral function for such patients. The inability to achieve adequate retention, stability, support and comfort due to the friability of healing tissues are the common problems associated with prosthetic treatment in the initial phase of treatment. This case report demonstrates a simple technique to overcome such difficulties by the use of soft lining materials in the delayed surgical obturator.
\end{abstract}

Key words: Hemimaxillectomy, Maxillofacial prosthesis, Soft liner, Surgical obturator

\section{Introduction}

$\mathrm{O}$ ral cancer is the eleventh most common cancer worldwide'. An obturator is a prosthesis which is placed after surgical resection of the affected maxillofacial structure. The primary purpose of an obturator is to separate the oral cavity from nasal cavity and form a pressure resistance seal against the oral mucosa to prevent leakage of air and fluid from nasal cavity. ${ }^{2}$ A surgical obturator also supports the soft tissues and prevents irritation of the mobile, non-cicatrized tissues and minimizes the scar contraction and facial disfigurement. ${ }^{3}$

The most common problems reported by patients wearing an obturator prosthesis include: leakage when swallowing foods, impairment of speech, chewing, swallowing, and pain ${ }^{4}$. The

\footnotetext{
*Corresponding Author

Dr. Prabhat Shrestha, Associate Professor

Department of Prosthodontics and Maxillofacial

Prosthetics, KIST Medical College and Hospital,

Lalitpur, Nepal.

E-mail: prabhatshrestha@gmail.com
}

frequency of problems is more in the early stages of tissue healing. Depending upon the nature of the defect, movement of the obturator varies and creates soreness and discomfort for the patient. These pressure sores are adjusted at the post insertion and subsequent follow-up appointments. ${ }^{5}$ Therefore, an effective treatment modality should be formed as a clinical protocol to increase comfort and reduce the number of recall visits. This case report demonstrates a simple and effective treatment modality using soft liners that may inherently increase patient satisfaction, comfort and decrease the number of adjustment appointments.

\section{Case Report}

A 39 years old woman was referred to the Department of Prosthodontics for the construction of palatal obturator prosthesis 8 days after surgical resection of the right maxilla along with orbital extension. The patient's original pathologic diagnosis was maxillary spindle cell variant rhabdomyosarcoma. She had an Aramany's class IV defect which included right palate with alveolar ridge including the 
upper right third molar upto the left central incisor teeth.

The primary complaint of the patient after surgery was difficulty in intake of food. There was remarkable limitation in opening of the oral cavity. Clinical examination revealed hollowness in the right palatal area with borders of surgical wound healing satisfactorily (fig 1).

The planned treatment was fabrication of palatal delayed surgical obturator so as to close the defect and restore the functions and aesthetics. Patient was explained about the treatment plan and following procedures were carried out:

The surgical borders were examined carefully and appropriate sized metallic perforated stock tray were selected. The hollow oro-nasal communication was packed with sterile cotton gauzes lubricated with petroleum jelly and a roof of oral cavity was created to contain the impression material. Impressions were then taken with irreversible hydrocollide impression material (Plastalgin Chromatic, Septodont Healthcare, India Pvt. Ltd.) and poured with dental stone type III (Kalstone, kalabhai Karson Pvt. Ltd., Mumbai, India) to make a cast (fig. 2).

The cast thus obtained was analyzed for the extent and the design of the obturator. Undercuts in the cast were blocked with dental plaster and a layer of $2 \mathrm{~mm}$ thickness of dental plaster was placed along the walls of the hollow defect. This was done to prepare space for the soft lining material. A piece of modelling wax was kept in the middle of the defect so as to make a hollow space in the obturator (fig. 3).

A 20 gauze stainless steel wire was used to fabricate continuous wrought wire clasps that engaged the facial infrabulge areas of the remaining maxillary teeth. An acrylic base plate was fabricated on the cast using autopolymerising acrylic resin (Trevalon, Dentsply, India Pvt. Ltd.) with proper incorporation of the clasps at dough stage of the resin in conventional method. Proper contours and bulks were made in the acrylic base plate in the facial flange and the alveolar ridge areas for proper lips and cheek support. After 24 hours the obturator was removed from the cast and modeling wax was removed. Excess of the acrylic resin was trimmed and polished.

The temporary obturator thus formed was disinfected and patient recall appointment was made. Try in of the prosthesis was done after examination of the oral cavity and the defects. Necessary adjustments were made. Finally, the obturator was relined with long term chairside soft lining material (Mollosil, Medicept Dental India Pvt. Ltd., India) following company instruction and reseated in the patient's mouth. Functional movements were performed to mould the soft lining material with the contours of the soft tissue (fig. $4 \& 5$ ).

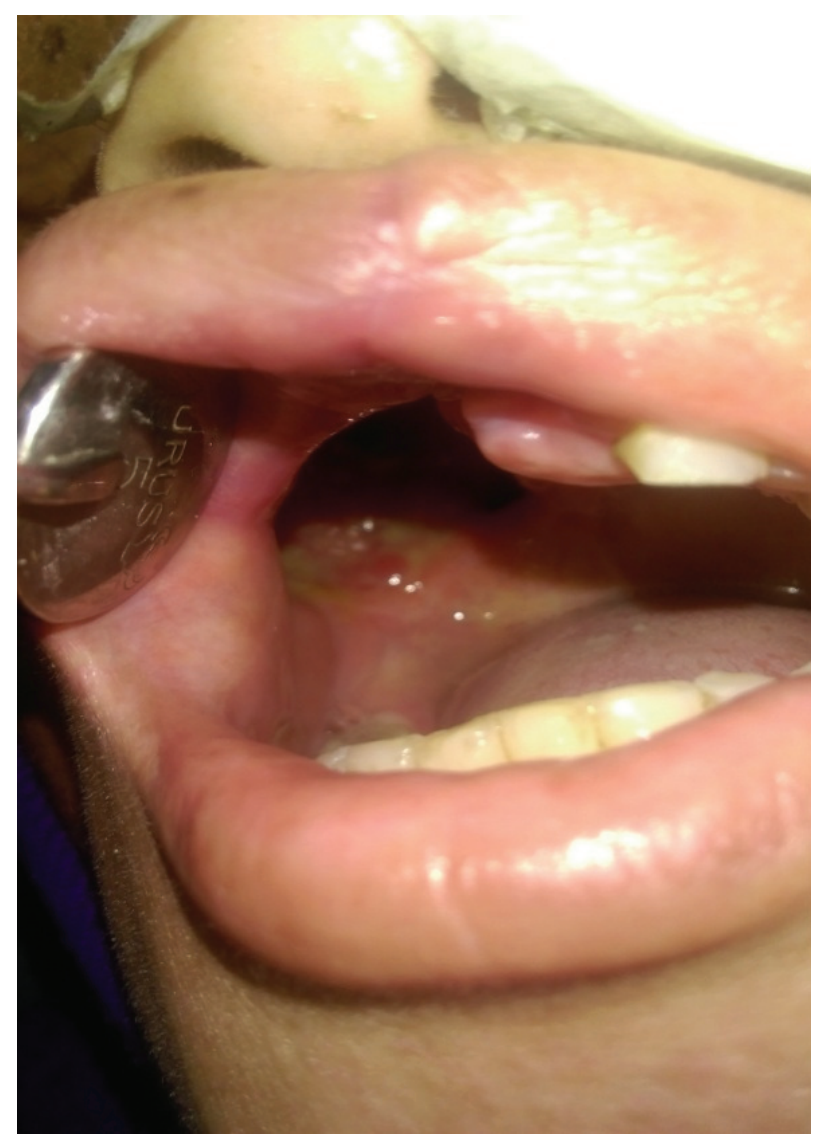

Figure 1: Right sided palatal defect in a 39 years old patient after hemi-maxillectomy. 


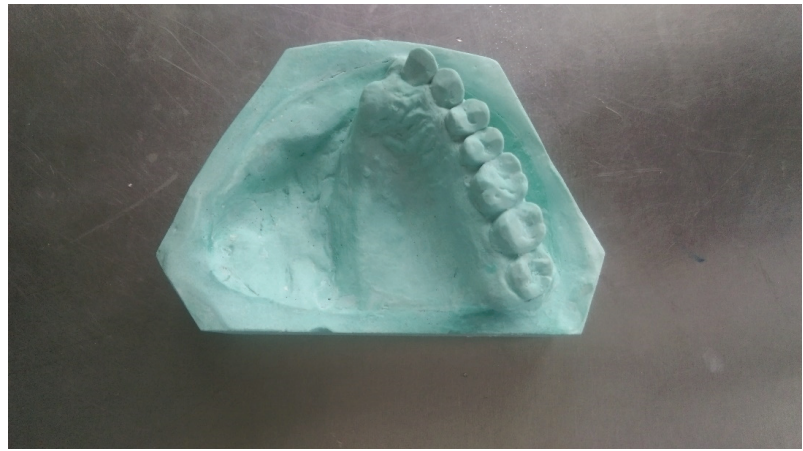

Figure 2: Maxillary cast of the patient showing the defect.

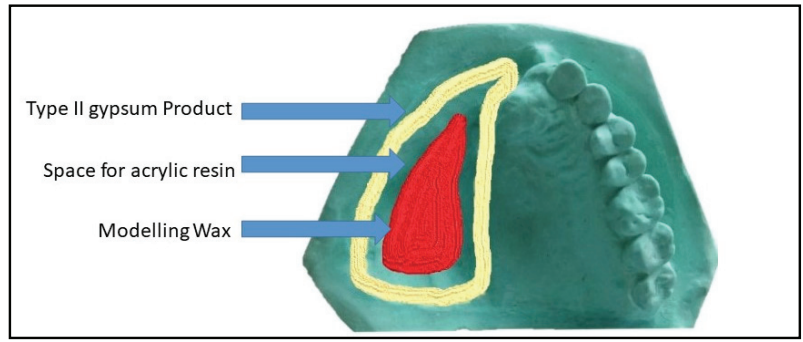

Figure 3: Diagrammatic representation of preparation of cast for fabrication of obturator

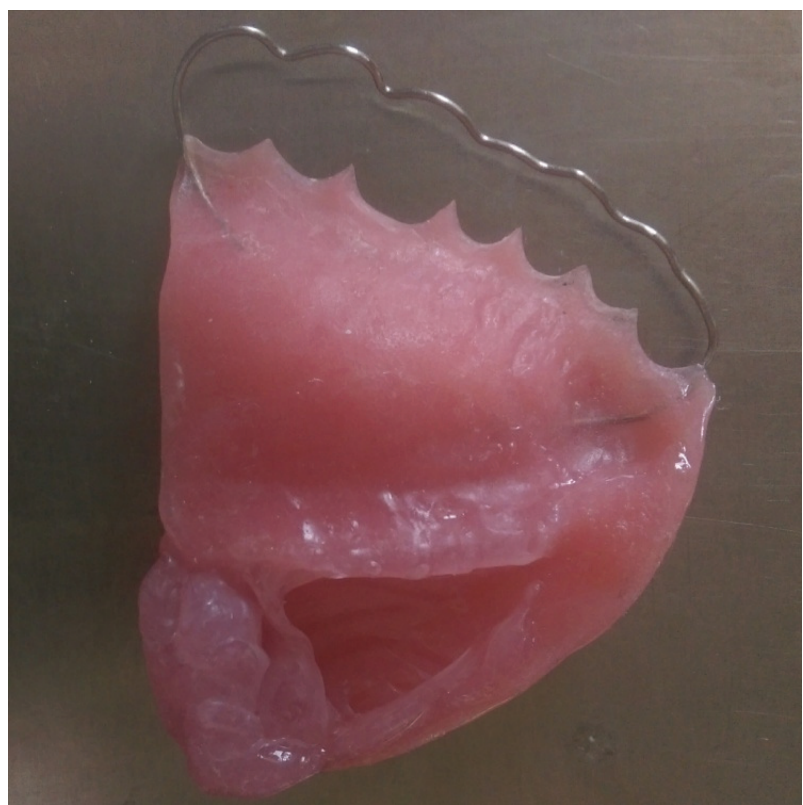

Figure 4: Self cure acrylic resin obturator with soft lining material.

\section{Discussion}

Delayed surgical obturators are usually fabricated from acrylic resin only. Modeling wax helped to create the vertical walls for support of soft lining material and also made the obturator hollow which will help to reduce its weight. ${ }^{5}$

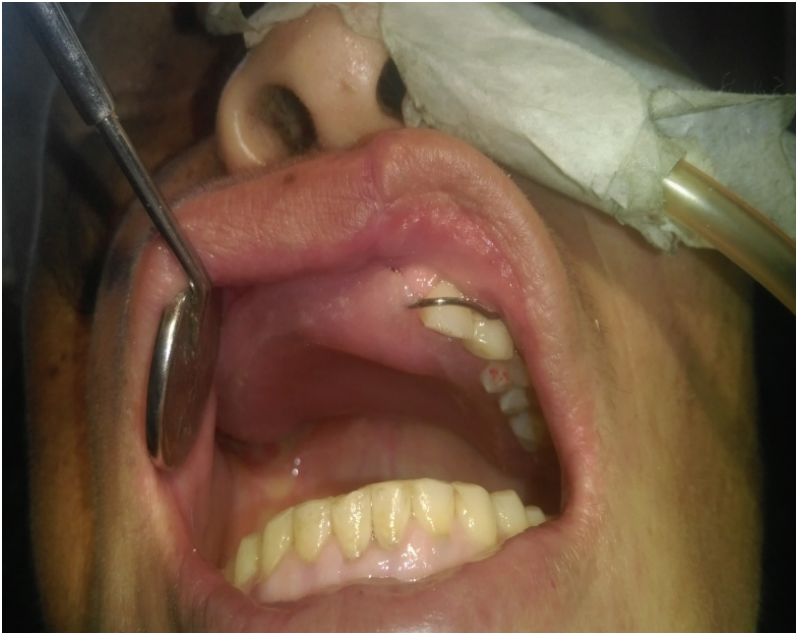

Figure 5: Obturator inserted in the mouth closing the defect.

The use of hard resin only may cause laceration and pressure sores on the friable, healing tissues. The patient has to visit the dentist for frequent follow-ups to adjust the obturator as the dimensional changes will continue to occur for at least 1 year secondary to scar contracture and further organization of the healing wound. The soft lining material allows to function as an impression material and become an integral part of the obturator prosthesis. ${ }^{6}$ This case report demonstrates a simple and effective treatment modality for achieving a tight contact with the tissue defect using soft liners that may inherently increase patient satisfaction, comfort and decrease the number of post insertion adjustment appointments.

\section{Conclusions}

The ease of this technique has made it suitable for both the clinician and the patient and can be incorporated in clinical practice to become a standard protocol to improve function and comfort while fabricating a delayed surgical obturator.

\section{References}

1. Ferlay J, Soerjomataram I, Dikshit R, Eser S, Mathers C, Rebelo M, et al. Cancer incidence and mortality worldwide: Sources, methods and major patterns in GLOBOCAN 2012. Int J Cancer. 2015;136(5):E359-86. 
2. Choudhury M, Shanmuganathan N, Padmanabhan T V., Swarup S, Grover M, Arumugam M. Rehabilitation of Post Surgical Maxillectomy Defects Using Interim Obturators-A Case Series. Indian J Surg Oncol. 2014;5(4):315-20.

3. Didier M, Laccoureye O, Brasnu D, Vignon M. New surgical obturator prosthesis for hemimaxillectomy patients. J Prosthet Dent [Internet]. 1993 May;69(5):520-3. Available from: http://linkinghub.elsevier.com/retrieve/ pii/002239139390163I

4. Peker K. Health-Related Quality of Life in Maxillectomy Patients Rehabilitated with Obturator Prostheses: A Literature Review.
In: Diagnosis and Management of Head and Neck Cancer [Internet]. InTech; 2017. Available from: http://www.intechopen.com/ books/diagnosis-and-management-of-headand-neck-cancer/health-related-quality-of-lifein-maxillectomy-patients-rehabilitated-withobturator-prostheses-a-li

5. Oh WS, Roumanas ED. Optimization of maxillary obturator thickness using a double-processing technique. J Prosthodont. 2008;17(1):60-3.

6. Jacob RF, Martin JW, King GE. Modification of surgical obturators to interim prostheses. J Prosthet Dent. 1985;54(1):93-5. 